\title{
Discovering naturally processed antigenic determinants that confer protective T cell immunity
}

\author{
Pavlo Gilchuk, ${ }^{1}$ Charles T. Spencer, ${ }^{1}$ Stephanie B. Conant, ${ }^{1}$ Timothy Hill, ${ }^{1}$ Jennifer J. Gray, \\ Xinnan Niu, ${ }^{1}$ Mu Zheng, ${ }^{1}$ John J. Erickson, ${ }^{1}$ Kelli L. Boyd, ${ }^{1}$ K. Jill McAfee, ${ }^{1}$ Carla Oseroff, ${ }^{2}$ \\ Sine R. Hadrup, ${ }^{3}$ Jack R. Bennink, ${ }^{4}$ William Hildebrand, ${ }^{5}$ Kathryn M. Edwards, ${ }^{6}$ \\ James E. Crowe Jr., 1,6 John V. Williams, 1,6 Søren Buus,7 Alessandro Sette,2 \\ Ton N.M. Schumacher, ${ }^{8}$ Andrew J. Link, ${ }^{1,9}$ and Sebastian Joyce ${ }^{1}$
}

\begin{abstract}
${ }^{1}$ Department of Pathology, Microbiology and Immunology, Vanderbilt University School of Medicine, Nashville, Tennessee, USA. ${ }^{2}$ Centre for Infectious Diseases, Allergy and Asthma Research, La Jolla Institute of Allergy and Immunology, La Jolla, California, USA. ${ }^{3}$ Centre for Cancer Immunotherapy, Department of Hematology, Herlev Hospital, Herlev, Denmark. ${ }^{4}$ Cellular Biology and Viral Immunology Section, Laboratory of Viral Diseases, National Institutes of Allergy and Infectious Diseases, NIH, Bethesda, Maryland, USA.

${ }^{5}$ Department of Microbiology and Immunology, University of Oklahoma Health Science Center, Oklahoma City, Oklahoma, USA. ${ }^{6}$ Department of Pediatrics, Vanderbilt University School of Medicine, Nashville, Tennessee, USA. ' 2 Laboratory of Experimental Immunology, University of Copenhagen, Copenhagen, Denmark. ${ }^{8}$ Division of Immunology, The Netherlands Cancer Institute, Amsterdam, The Netherlands. ${ }^{9}$ Department of Biochemistry, Vanderbilt University School of Medicine, Nashville, Tennessee, USA.
\end{abstract}

$\mathrm{CD8}^{+} \mathrm{T}$ cells (TCD8) confer protective immunity against many infectious diseases, suggesting that microbial TCD8 determinants are promising vaccine targets. Nevertheless, current $T$ cell antigen identification approaches do not discern which epitopes drive protective immunity during active infection - information that is critical for the rational design of TCD8-targeted vaccines. We employed a proteomics-based approach for large-scale discovery of naturally processed determinants derived from a complex pathogen, vaccinia virus (VACV), that are presented by the most frequent representatives of four major HLA class I supertypes. Immunologic characterization revealed that many previously unidentified VACV determinants were recognized by smallpox-vaccinated human peripheral blood cells in a variegated manner. Many such determinants were recognized by HLA class I-transgenic mouse immune TCD8 too and elicited protective TCD8 immunity against lethal intranasal VACV infection. Notably, efficient processing and stable presentation of immune determinants as well as the availability of naive TCD8 precursors were sufficient to drive a multifunctional, protective TCD8 response. Our approach uses fundamental insights into $\mathrm{T}$ cell epitope processing and presentation to define targets of protective TCD8 immunity within human pathogens that have complex proteomes, suggesting that this approach has general applicability in vaccine sciences.

\section{Introduction}

Vaccination is an effective approach to prevent infectious diseases. The majority of current vaccines consists of live, attenuated, or inactivated pathogens that were developed in the past by empirical approaches (1). Understanding vaccine-mediated correlates of immunity is critical for the rational design of novel pathogen-free vaccines against diseases caused by complex pathogens - such as malaria, tuberculosis, or HIV/AIDS, for which no effective vaccine currently exists - or to improve the safety and/or efficacy of existing vaccines (1-6).

$\mathrm{CD}^{+} \mathrm{T}$ cells (TCD8) play a critical role in conferring protective immunity against many infectious diseases, including those caused by respiratory viruses (7-11), cytomegalovirus (12), HIV (13), Plasmodium spp. $(14,15)$, and M. tuberculosis $(16,17)$. A key role for TCD8 immunity has also been established for the safety of smallpox vaccination in nonhuman primates (18) and humans (19). Therefore, microbial $\mathrm{T}$ cell epitope-based vaccines can complement current efforts to develop safe and effective antibody-targeted subunit vaccines.

Identification of immune TCD8 targets is a complicated task, because of MHC class I polymorphism and the ability to generate

Conflict of interest: The authors have declared that no conflict of interest exists. Citation for this article: J Clin Invest. 2013;123(5):1976-1987. doi:10.1172/JCI67388. numerous potential epitopes from complex microbial proteomes for TCD8 appraisal. Computational epitope prediction in combination with TCD8 recognition assays is one solution to this quandary (20). This approach has identified numerous immune epitopes from different pathogens (http://www.iedb.org/). In addition, to elucidate the protective potential of TCD8 immunity, different correlates - such as the response magnitude (21), epitope immunogenicity, binding affinity (22), p/MHC stability (23), and recognition potency (24) as well as the ability to generate multifunctional effector response (25) and long-lived memory response (26) - have been comprehensively evaluated.

Vaccinia virus (VACV; $\sim 200$ proteins) is widely used as a model to study TCD8 responses to pathogens with complex proteomes. Interrogation of computationally predicted epitopes revealed that humans and mice recognize numerous epitopes by VACV-immune TCD8; humans recognize these epitopes in a variegated, i.e., partially overlapping, manner (27-31). Nevertheless, due to the current limitations of bioinformatics prediction approaches, identification of viral determinants that are efficiently presented in vivo and initiate protective TCD8 responses remains a challenge. In addition, the complexity of antimicrobial TCD8 responses (27-31) severely limits the screening of even known immune determinants for protective efficacy. Hence, only limited preclinical mouse studies have addressed 

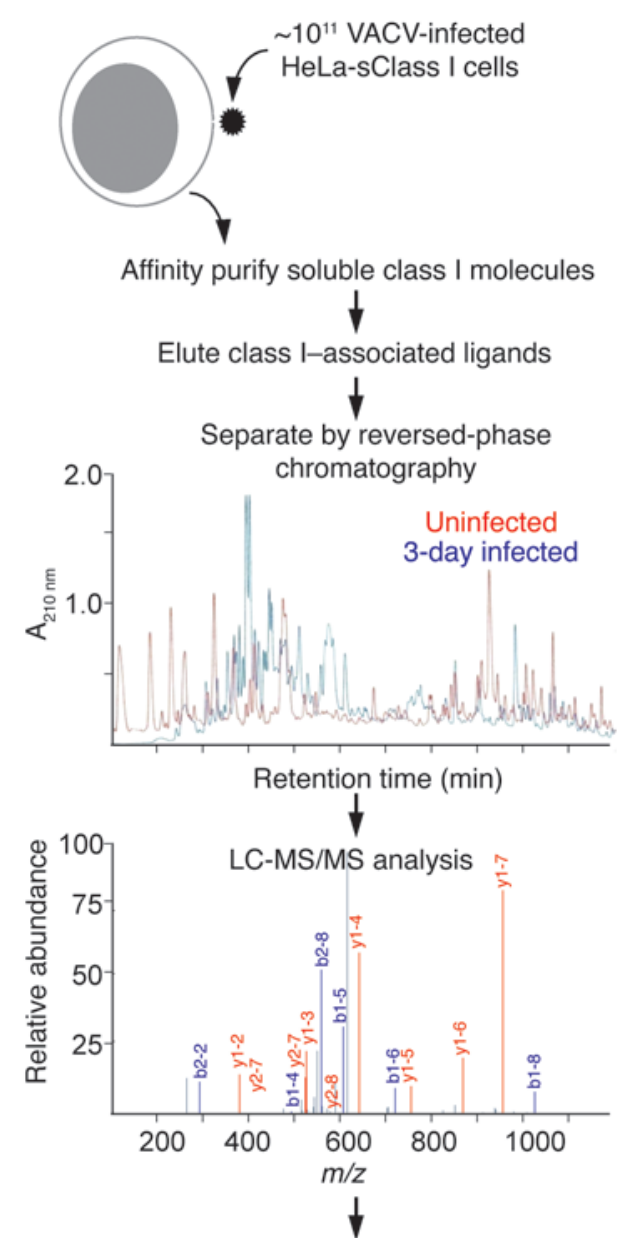

Analyze by SEQUEST

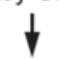

$\mathrm{A} 2$ and $\mathrm{B} 7$ binding motif

A2: P2L/I/M/V; PQL/N

$\mathrm{B} 7: \mathrm{P} 2 \mathrm{P} ; \mathrm{P} \Omega \mathrm{L} / \mathrm{I} / \mathrm{M} / \mathrm{V}$

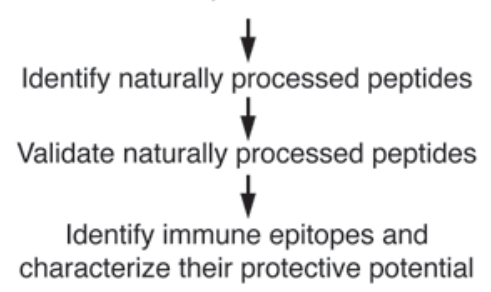

the protective potential of individual antigenic determinants, which were mostly focused on a few rapidly detectable specificities (31-34). Therefore, the actual array of critical antigenic determinants driving protective TCD8 responses during a natural infection or after vaccination remains unknown. Furthermore, it has been noted that the computational approach has underestimated the breadth of VACV-derived TCD8 epitopes (35), necessitating a reevaluation of TCD8 epitope presentation by alternative approaches.

As alternatives to algorithm-based epitope prediction, mass spectrometry-based proteomics approaches to sequence peptides eluted from HLA class I molecules have been used to identify VACV epitopes $(32,36,37)$. A significant advantage to this approach is that it directly informs whether an antigenic determinant is natu-

\section{Figure 1}

Schema of the approach for characterizing naturally processed, HLA class I-restricted TCD8 epitopes. HeLa cells engineered to secrete soluble HLA class I molecules (HeLa-sClass I cells) are inoculated with VACV. Secreted class I molecules are immunoaffinity purified from day 1,2 , and 3 p.i. culture supernatant using W6/32 mAb-bound protein A-Sepharose columns. Associated peptides are isolated and separated by reversed-phase HPLC, and their amino acid sequence was determined by LC-MS/MS. Those peptides derived from VACV that conformed to the canonical $\mathrm{A} 2$ and $\mathrm{B} 7$ binding motifs are validated using the corresponding synthetic peptide spectra and considered naturally processed class I-restricted determinants. That these are TCD8 epitopes is ascertained by functional assays and dual $\mathrm{p} / \mathrm{class}$ I tetramer staining. Additionally, the protective potential of the TCD8 epitopes is tested in peptide vaccination and a respiratory VACV challenge model.

rally processed, thereby narrowing the search for potential vaccine targets to those determinants that are efficiently presented during an infection (38). While only a few such epitopes have been evaluated for immune recognition $(32,37)$, none were ever interrogated for their protective potential. Therefore, it remains unclear whether the proteomics approach is superior for TCD8 epitope discovery over currently existing computational approaches and whether eluted peptides inform relevant vaccine targets.

In this study, we used VACV as a model, because a critical role has been attributed to TCD8 in conferring protective immunity against lethal respiratory VACV infection $(10,11)$. Using data from a comprehensive proof-of-principle study for the identification of potent TCD8 targets from a complex virome, we report large-scale discovery and immunologic characterization of naturally processed antigenic determinants of VACV that are presented by five frequent HLA class I allotypes that represent four major class I supertypes. Additionally, we provide direct evidence that naturally processed epitopes inform targets for TCD8based protective immunity. Our strategy, in which fundamental insights into $\mathrm{T}$ cell epitope processing and presentation were used to define targets of protective TCD8 immunity, should have general applicability in vaccine sciences.

\section{Results}

Numerous VACV-derived determinants are naturally processed and presented by HLA class I molecules. Recognition of infected cells by TCD8 relies on efficient presentation of microbial determinants. We used a proteomics approach coupled with functional studies to identify and characterize naturally processed HLA class I-restricted determinants presented during active poxvirus infection (Figure 1). HeLa cells expressing soluble HLA-A*02;01 (sA2), -B*07;02 (sB7), -B35;01 (sB35), -A01;01 (sA1), or -B45;01 (sB45) molecules were infected with VACV, and associated peptides were eluted from class I molecules and fractionated by reversed-phase chromatography. In-line microcapillary HPLC-electrospray ionization tandem mass spectrometry (LC-MS/MS) of each eluted peptide fraction resulted in the acquisition of a large number of spectra (data not shown). Those that contained 9-11 amino acid residues and conformed to the canonical HLA-A*02;01 (A2), - $\mathrm{B}^{*} 07 ; 02$ (B7), -B35;01 (B35), -A01;01 (A01), and -B45;01 (B45) binding motifs were compared against both human and vaccinia viral proteomes (see Methods). We limited the analysis to peptides that contain 9-11 residues, because the large majority conforms to this length while a small fraction contains longer peptides. 
Table 1

Summary of immune epitopes identified in smallpox-vaccinated B7 volunteers

\begin{tabular}{|c|c|c|c|c|c|}
\hline \multicolumn{6}{|c|}{ Volunteers ${ }^{\mathrm{A}}$} \\
\hline Peptide sequence & V003 & V111 & V150 & V154 & 155 \\
\hline \multicolumn{6}{|c|}{ Novel immune epitopes identified in this study } \\
\hline FPYEGGKVF & & & & $456^{\mathrm{B}}$ & \\
\hline FPRSMLSIF & 222 & & & & \\
\hline SPSNHHILL & & & 291 & $823^{C}$ & \\
\hline FPKNDFVSF & & $534 \mathrm{C}$ & & $539 \mathrm{c}$ & \\
\hline RPRDAIRFL & & $367^{c}$ & & & \\
\hline RPNQHHTIDL & & $576^{c}$ & & & \\
\hline APASSLLPAL & & & & 392 & \\
\hline FPSVFINPI & & & & 332 & \\
\hline VPITGSKLIL & & & 158 & 9,074 & 278 \\
\hline YPSNKNYEI & & & 144 & 8,701 & 238 \\
\hline LPSNVEIKAI & & & & 1,317 & \\
\hline \multicolumn{6}{|l|}{ IPKYLEIEI } \\
\hline NPSKMVYALL & & & 291 & 1,725 & \\
\hline NPSVLKILL & & & & & \\
\hline \multicolumn{6}{|c|}{ Known immune epitopes also found as naturally processed in this study } \\
\hline RPSTRNFFEL & & & 624 & $2,235^{c}$ & \\
\hline \multicolumn{6}{|c|}{ Known immune epitopes not found as naturally processed in this study } \\
\hline TVADVRHCL & & $1,226^{c}$ & 291 & $2,588^{C}$ & 165 \\
\hline RPMSLRSTII & & $985^{C}$ & & & \\
\hline MPAYIRNTL & & $157 \mathrm{C}$ & & & \\
\hline HPRHYATVM & $257^{c}$ & & & & \\
\hline
\end{tabular}

AThe HLA class I allotype for each donor is provided in Supplemental Table 2. BThe numbers of spot-forming cells over background per million PBMCs that secrete IFN- $\gamma$ in response to individual peptides are presented; responses are considered positive only if spot-forming cells per million PBMCs were at least 100 over background.

cEpitopes also found as positive in tetramer binding assay.

The comparative proteome searches identified 581 sA2and 132 sB7-associated peptides that were expressed only by VACV-infected but not uninfected cells; of those, 109 A2- and 65 B7-restricted peptide sequences correlated with the MS/MS spectra's fragment ion data with high confidence (see Methods, Supplemental Figure 1, and Supplemental Table 1; supplemental material available online with this article; doi:10.1172/ JCI67388DS1) and, hence, were selected for synthesis and further study. Similar analysis identified numerous naturally processed VACV peptides eluted from sA1, sB35, and sB45 molecules as well (our unpublished observations).

Mass spectrometry was performed with a low-resolution instrument (LTQ ion trap), which made data analysis challenging. Hence, synthetic peptides corresponding to the amino acid sequences of naturally processed peptides were analyzed by LC-MS/MS for spectral validation of each eluted peptide. For this purpose, the spectrum of the eluted peptide was qualitatively compared to that of the corresponding synthetic peptide. We found that the MS/MS spectra of most of the eluted peptides were similar to those of the corresponding synthetic peptides (Supplemental Figure 1 and data not shown), confirming the identity of the naturally processed peptides.

Comparison to the VACV proteome revealed that the naturally processed peptides were derived from all functional and kinetic classes of VACV proteins, with multiple determinants emerging from several ORFs (Supplemental Table 1), consistent with previous reports (28). Importantly, many known immune epitopes reported from algorithm-predicted determinants were also identified as naturally processed (Supplemental Table 1), indicating that the proteomics approach can be used for the discovery of TCD8 targets. Critically, however, we also identified dozens of determinants that would not be predicted to be TCD8 epitopes based upon the bioinformatics approach alone, many of which we validated as bona fide TCD8 targets (see below). Thus, we found from this thorough, largescale analysis that, during VACV infection, a large and diverse array of naturally processed viral determinants was presented by representative members of the four most frequent HLA class I supertypes - i.e., A2 (A2), B7 (B7, B35), A1 (A1), and B44 (B45).

Many novel naturally processed determinants are recognized by human TCD8. We next ascertained whether viral peptides eluted from infected HeLa cells expressing soluble HLA class I molecules inform relevant targets for immune recognition during a natural infection in humans. Hence, the TCD8 response to a peptide panel (defined below) was evaluated using PBMCs from smallpox-vaccinated volunteers: 6 B7or 7 A2-positive volunteers, either prior to (3 of each HLA type) or after DryVax vaccination (Supplemental Table 2). Because the frequency of VACV-specific TCD8 in humans is very low after vaccination $(39,40)$, epitope-specific TCD8 from PBMCs were expanded ex vivo prior to the assay. The peptide panel interrogated consisted of $83 \mathrm{~B} 7$ - and $152 \mathrm{~A} 2$-restricted determinants (Supplemental Table 3); 65 B7- and 109 A2-restricted peptides were novel determinants found in this study, and the remainder was selected from previous reports (http://www.iedb.org/). Two complementary assays - IFN- $\gamma$ ELISpot and dual fluorochrome-conjugated $\mathrm{p} /$ class I tetramer staining approach - were used to determine the activity of the peptide panel. Data from the IFN- $\gamma$ ELISpot assay revealed that each vaccinated individual showed only a partially overlapping, variegated recognition of the naturally processed determinant panel (Table 1).

To ascertain whether the IFN- $\gamma$ response to the naturally processed peptides was TCD8 derived, a panel of $\mathrm{p} /$ class I tetramers was prepared and their binding to ex vivo-expanded PBMCs was quantified. $\mathrm{p} /$ class I monomers only formed with $\sim 55 \%$ of the total peptides due to poor binding of some low-affinity peptides to the cognate class I molecule (see $t_{1 / 2}$ in Supplemental Table 1 and data not shown), thereby narrowing the TCD8 specificities that could be probed. $\mathrm{p} /$ class I tetramer staining confirmed that the IFN- $\gamma$ response was indeed generated by B7- or A2-restricted TCD8 (Table 1, Supplemental Figure 2, and Supplemental Table 4). Notably, immunologic screening of naturally processed peptides revealed that many novel immune epitopes were recognized by human PBMCs obtained from smallpox vaccinees in addition to those reported from algorithm-based approaches (Table 1 and Supplemental Table 4). Most importantly, these results indicate that the eluted viral determinants identified by our proteomics approach inform relevant targets for TCD8 recognition.

TCD 8 from HLA class I-transgenic mice recognize naturally processed determinants. We used a HLA class I-transgenic mouse model to define the qualitative and quantitative features of VACV-specific TCD8 response reactive to the eluted, naturally processed 
A VACV challenge, Analyze i.p.

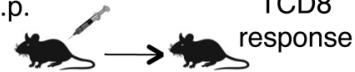

d $0 \quad$ d 7

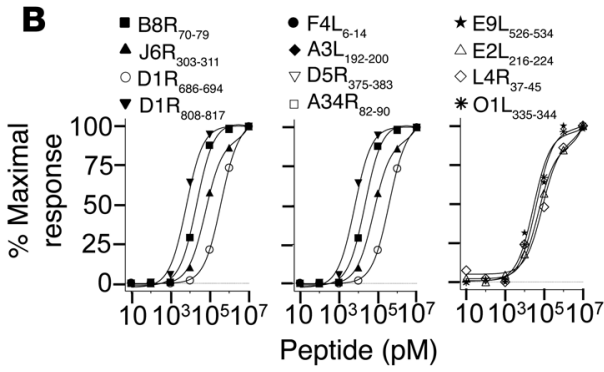

D

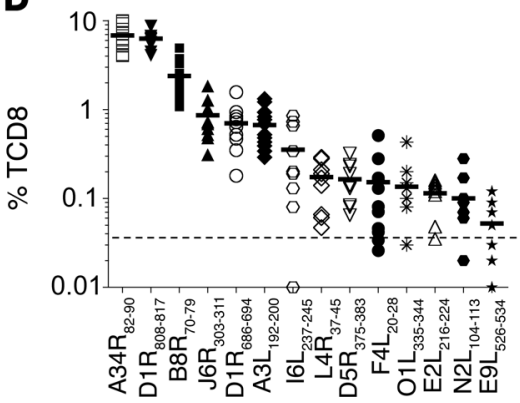

C
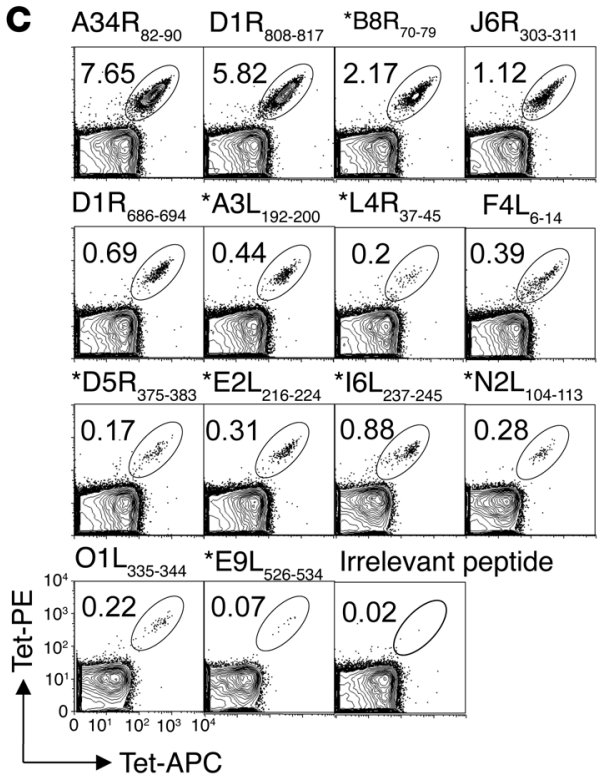

Figure 2

Tracking VACV-specific TCD8 responses in B7 transgenic mice. (A) Experimental design. B7tg mice were inoculated i.p. with $2 \times 10^{5}$ pfu VACV, and splenic TCD8 response was characterized on day 7. (B) Dosedependent IFN- $\gamma$ response by VACV-immune splenocytes measured by epitope titration in the ELISpot assay. Data represent the mean of triplicate wells and are representative of 2 independent experiments $(n=5)$. (C) TCD8 responses detected using p/B7.2 tetramers on VACV-immune splenocytes. Data are representative of at least 3 independent experiments using pooled spleens $(n=5-10)$. Numbers indicate the percentage of $\mathrm{CD}^{+} \mathrm{p} / \mathrm{B} 7.2$ tetramer ${ }^{+}$cells. The asterisks indicate novel immune epitopes. (D) VACV-specific TCD8 hierarchy defined by p/class I tetramer staining of VACV-immune splenocytes. Cumulative data from 3 to 13 independent experiments $(n=5-8)$. Each symbol represents TCD8 frequency from 1 experiment. The dotted line indicates assay background defined by irrelevant HMPV-derived TCD8 epitope/B7 tetramer binding (68). Horizontal bars indicate the mean.

determinants identified above. Splenocytes from acutely infected $\mathrm{B} 6-K^{0} D^{0} ; B^{*} 0702^{\text {tg }}\left(\mathrm{B} 7^{\mathrm{tg}}\right)$ and $\mathrm{B} 6-K^{0} D^{0} ; A^{*} 0201^{\text {tg }}\left(\mathrm{A} 2^{\mathrm{tg}}\right)$ mice functionally responded to 12 out of $83 \mathrm{~B} 7$ - and 15 out of 152 A2-restricted epitopes in the ELISpot assay (Figure 2, A and B, Supplemental Figure 3, and Supplemental Figure 4A). Within the same VACV-immune splenocytes, $\mathrm{p} /$ class I tetramers specifically identified 14 B7- and 18 A2-restricted epitopes, demonstrating a strong concordance between the two assays (Figure 2C and Supplemental Figure 4, B and C). Notably, this screen revealed many novel TCD8 epitopes, in addition to those identified by algorithm-based approaches (Table 2 and Supplemental Table 5).

Akin to TCD8 responses to other pathogens (41), those against $\mathrm{VACV}$ in $\mathrm{B} 7^{\mathrm{tg}}$ mice followed a reproducible hierarchy such that certain specificities were dominant and others were subdominant, which we defined as $>1 \%$ or $<1 \%$ of $\mathrm{p} /$ class I tetramer-positive TCD8, respectively (Figure 2D and Supplemental Figure 4D). To confirm that subdominant TCD8 specificities detected were indeed antigen specific and not cross-reactive, splenocytes from VACV-immune and naive B $7^{\mathrm{tg}}$ mice were expanded ex vivo with individual antigenic peptides in the presence of recombinant human IL-2, followed by staining with p/B7.2 tetramers on day 7 postinoculation (p.i.). Robust expansion of epitope-specific TCD8 from VACV-immune splenocytes but not from naive splenocytes was observed (Supplemental Figure 5), indicating that all antigen-specific reactivity detected (Figure 2 ) was specifically elicited in response to $\mathrm{VACV}$ in vivo.

In an independent approach, we assessed whether the TCD8-reactive peptides discovered by proteomics are actual epitopes generated through processing and presentation from cognate VACV proteins in vivo. For this purpose, B $7^{\mathrm{tg}}$ mice were immunized with individual VACV-derived proteins containing targeted epitope sequences. Nine recombinant proteins containing the ten immune determinants (two of which are D1R derived; Supplemental Figure 6) were produced in E. coli and purified. B $7^{\mathrm{tg}}$ mice were primed and boosted (i.p.) with individual proteins formulated with the natural killer $\mathrm{T}$ cell ligand $\alpha$-galactosylceramide (Supplemental Figure 7A), which is known to function as an adjuvant (42-45). All proteins were immunogenic when formulated with $\alpha$-galactosylceramide and induced a robust systemic response, as judged by detection of epitope-specific TCD8 with p/B7.2 tetramers at day 6 after boost (Supplemental Figure 7B, Supplemental Figure 8 , and data not shown). Moreover, such protein-elicited TCD8 functionally responded to restimulation with antigenic peptide, which induced the expression of intracellular IFN- $\gamma$ and mobilized CD107a - a marker for degranulation - to the plasma membrane (Supplemental Figure 7C). Hence, we conclude that all the tested immune determinants are processed and presented from the cognate antigenic proteins in vivo.

To determine whether the stability of epitope presentation influenced TCD8 recognition, the half-life of each of the naturally processed $\mathrm{p} /$ class I complexes was quantified. We found that most of the immune determinants identified in this study were stably presented by their cognate class I molecules (Table 2 and Supplemental Tables 1 and 5). Together the data suggest that the antigenic determinants identified here are potential targets for protective TCD8 responses during a natural infection.

Individual TCD8 specificities targeting naturally processed epitopes exhibit similar phenotype, functional competence, and homing pattern. The immunologic rationale for the selection of potent vaccine targets among numerous determinants should rely on their ability to elicit 
Table 2

Summary of immune epitopes identified in B7 transgenic mice

\begin{tabular}{|c|c|c|}
\hline Sequence $^{A}$ & ORF & $t_{1 / 2}(h)$ \\
\hline \multicolumn{3}{|c|}{ Novel immune epitopes } \\
\hline FPKNDFVSFB & $\mathrm{B} \mathrm{R}_{70-79}$ & 5.2 \\
\hline SPSNHHILLB & $\mathrm{A} 3 \mathrm{~L}_{192-200}$ & 5.46 \\
\hline LPKEYSSEL ${ }^{B}$ & $\mathrm{D} \mathrm{R}_{375-383}$ & 7.44 \\
\hline FPYEGGKVFB & E9L $526-534$ & 6.17 \\
\hline RPRDAIRFL ${ }^{B}$ & $\mathrm{E} 2 \mathrm{~L}_{216-224}$ & 4.81 \\
\hline FPRSMLSIFB & $\mathrm{L}_{4} \mathrm{R}_{37-45}$ & 6.08 \\
\hline RPNQHHTIDLB & $N 2 L_{104-113}$ & 4.73 \\
\hline FPTNTLTSIB & $16 \mathrm{~L}_{237-245}$ & 1.52 \\
\hline \multicolumn{3}{|c|}{$\begin{array}{l}\text { Known immune epitopes also found as naturally } \\
\text { processed in this study }\end{array}$} \\
\hline RPSTRNFFEL ${ }^{B}$ & $\mathrm{D} 1 \mathrm{R}_{808-817}$ & 2.93 \\
\hline LPRPDTRHLB & $\mathrm{A} 34 \mathrm{R}_{82-90}$ & 1.52 \\
\hline \multicolumn{3}{|c|}{$\begin{array}{l}\text { Known immune epitopes not found as naturally } \\
\text { processed in this study }\end{array}$} \\
\hline MPAYIRNTLB & $\mathrm{J} 6 \mathrm{R}_{303-311}$ & 5.44 \\
\hline HPRHYATVMB & ${\mathrm{D} 1 \mathrm{R}_{686-694}}$ & 6.47 \\
\hline APNPNRFVIB & $\mathrm{F} 4 \mathrm{~L}_{6-14}$ & 8.81 \\
\hline RPMSLRSTIIB & $01 \mathrm{~L}_{335-344}$ & 5.31 \\
\hline
\end{tabular}

AIdentified by IFN- $\gamma$ ELISpot assay. ${ }^{B}$ Epitopes also found as positive in tetramer binding assay.

multifunctional, protective responses. Hence, to identify measurable qualities that can predict their protective capacity, $\mathrm{B} 7^{\mathrm{tg}}$ mice were inoculated with VACV and, on day 7, the functional potency, clonal diversity, and tissue distribution were determined for splenic immune TCD8 recognizing the 10 B7-restricted VACV determinants. TCD8 responses against the 10 determinants were nonoverlapping (Supplemental Figure 9, A and B) and accounted for about one-half of the overall TCD8 response to VACV (Supplemental Figure 10A). Analysis of biomarkers associated with antigen exposure revealed effector $\left(\mathrm{CD} 44^{\mathrm{hi}} \mathrm{CD} 62 \mathrm{~L}^{\text {lo }} \mathrm{GzmB}{ }^{+} \mathrm{KLRG} 1^{\text {hi }}\right)$ and memory (CD44hi CD62L hi CD127 hi) phenotypes within both dominant and subdominant specificities (Figure 3, A and B). We also quantified 4 TCD8 effector mediators, because the protective efficacy of $\mathrm{T}$ cell-based vaccines was previously correlated with the frequency of multifunctional effectors $(25,46)$. Remarkably, intracellular IFN- $\gamma$, TNF- $\alpha$, and IL- 2 synthesis and cytolytic granule release (judged by surface CD107a mobilization) upon ex vivo restimulation with antigenic peptide were proportional to the frequency of a given TCD8 (Figure 3, C and D). These results suggest similar functional competence of dominant and subdominant TCD8 specificities elicited in the primary response to VACV. Furthermore, each of the analyzed immune determinants was recognized by a polyclonal $\mathrm{T}$ cell receptor repertoire (Figure 3E and Supplemental Figure 10B). Last, the 6 most frequent TCD8 specificities were proportionally distributed to all tissues in frequencies reminiscent of the hierarchy observed in the spleen (Figure 3F and Supplemental Figure 10C).

Collectively, our results indicate that the different TCD8 specificities generated against naturally processed VACV determinants exhibit similar phenotype, functional competence, and homing pattern irrespective of their dominance hierarchy. Thus, efficient recruitment of virtually any precursor TCD8 (pTCD8) that recognize naturally processed VACV determinant has the potential to generate a protective response.
Most immune TCD8 specificities elicited against efficiently presented epitopes contribute to protection against VACV. Efficient recruitment of naive antigen-specific PTCD8 that can evolve into effector and memory cells is required to generate protective immunity. Hence, to determine the extent of TCD8 recruitment upon VACV infection, we compared frequencies of naive and immune TCD8 recognizing individual naturally processed epitopes. For this purpose, pTCD 8 against the 10 characterized immune epitopes were enumerated in naive B $7^{\mathrm{tg}}$ mice after successive TCD8 and $\mathrm{p} / \mathrm{B} 7.2$ tetramer-based enrichments (Supplemental Figure 11). We found that the VACV epitope-specific pTCD8 frequencies ranged from approximately 126 (E2 $\left.\mathrm{L}_{216-224}\right)$ to approximately 6,104 $\left(\mathrm{L}_{4} \mathrm{R}_{37-45}\right)$ per mouse, which corresponds to 17 and 731 pTCD 8 per $10^{6}$ naive TCD8, respectively (Figure 4). Of the 10 epitopes, only 3 - namely,

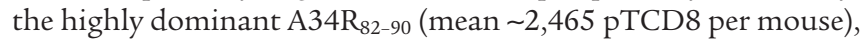

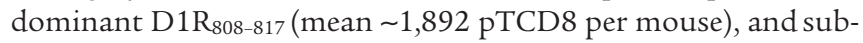
dominant $\mathrm{L}_{4} \mathrm{R}_{37-45}$ (mean $\sim 6,104$ pTCD8 per mouse) - were higher than those reported for the most frequent PTCD8 - e.g., MCMV M45:Db $\left(\sim 1,500\right.$ pTCD8 per mouse) and VACV B8R:K $\mathrm{K}^{\mathrm{b}}(\sim 1,400$ pTCD8 per mouse) epitopes (47-51). B $7^{\mathrm{tg}}$ mice contain only about a third of the total TCD $8\left(\sim 7 \times 10^{6}\right.$ to $8 \times 10^{6}$ TCD 8 per mouse; data not shown) ordinarily generated by C57BL/ 6 mice $\left(\sim 17 \times 10^{6}\right.$ TCD8 per mouse; ref. 51), perhaps because the former expresses only one class I allele, as they do not express mouse H2 class I molecules. Hence, the normalized pTCD8 frequency per $10^{6}$ TCD8 appears abnormally high. Notable, for 3 dominant $\left(A_{34} R_{82-90}\right.$,

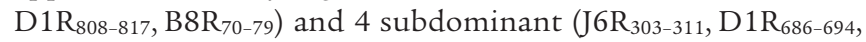
$\left.\mathrm{A} 3 \mathrm{~L}_{192-200}, \mathrm{E} 2 \mathrm{~L}_{216-224}\right) \mathrm{TCD} 8$ specificities, we found that the pTCD8 frequency corresponded to the effector hierarchy established during primary VACV infection (Figure 2D and Figure 4). This result suggests proportional expansion of the corresponding naive pTCD8 during infection.

Surprisingly, the naive pTCD8 frequency for the 3 remaining subdominant determinants $\left(\mathrm{L}_{4} \mathrm{R}_{37-45}, \mathrm{D} \mathrm{R}_{375-383}, \mathrm{~F}_{4} \mathrm{~L}_{6-14}\right)$ was not concordant with the magnitude of the primary TCD8 response (Figure 2D and Figure 4), suggesting that these determinants are less potent TCD 8 targets. To test this prediction, all 10 immune epitopes were individually formulated with anti-CD40 antibody plus poly(I:C) as adjuvant (see Methods) and used to immunize mice, an approach reported to efficiently elicit TCD8 responses (52). Tracking of blood TCD8 with p/B7.2 tetramers revealed that some determinants, including the dominant $\mathrm{B}_{8} \mathrm{R}_{70-79}$ epitope, were not immunogenic, even after repeated vaccinations (data not shown). This lack of immunogenicity, previously described for some $\mathrm{H} 2 \mathrm{~K}^{\mathrm{b}}$-restricted immune determinants (22), was not predicted by the frequency of naive or VACV-immune TCD8. Critically, nonetheless, when mice were primed with in vitromaturated, peptide-loaded DCs, all 10 epitopes elicited robust epitope-specific secondary responses, which accounted for $4 \%$ to $92 \%$ of total blood TCD8 (Figure 5, A and B). Thus, our results suggest that many of the available precursor specificities, including those for subdominant TCD8, have the potential to be efficiently recruited to the immune response when the cognate antigenic determinant is efficiently presented.

We next determined the protective potential of individual TCD8 specificities recognizing naturally processed determinants. For this, the above epitope-vaccinated mice were lethally challenged by i.n. VACV; these mice were observed during the next 6 days for morbidity and weight loss, and, on day 6 after challenge, lung viral burdens were measured as well. To ensure elicitation of robust 
technical advance

A

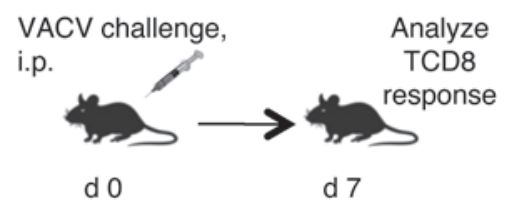

C
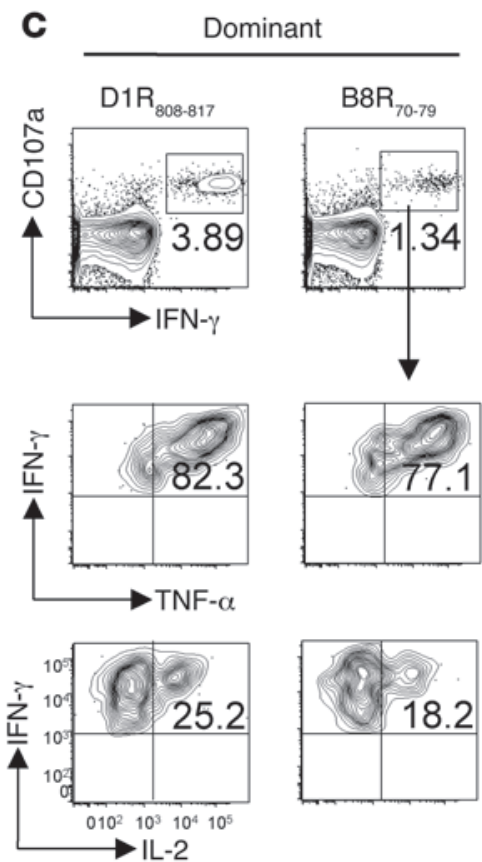

D

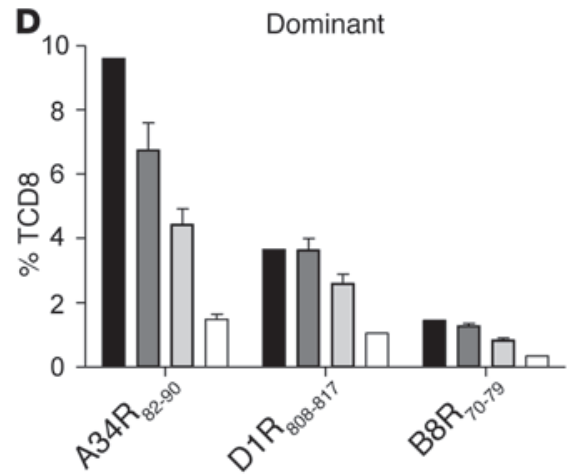

E Dominant $\quad$ Subdominant

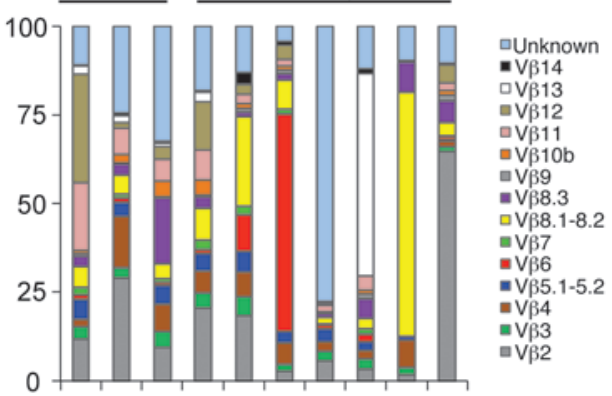

B

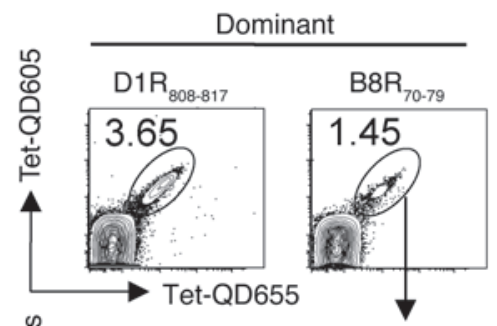

Subdominant
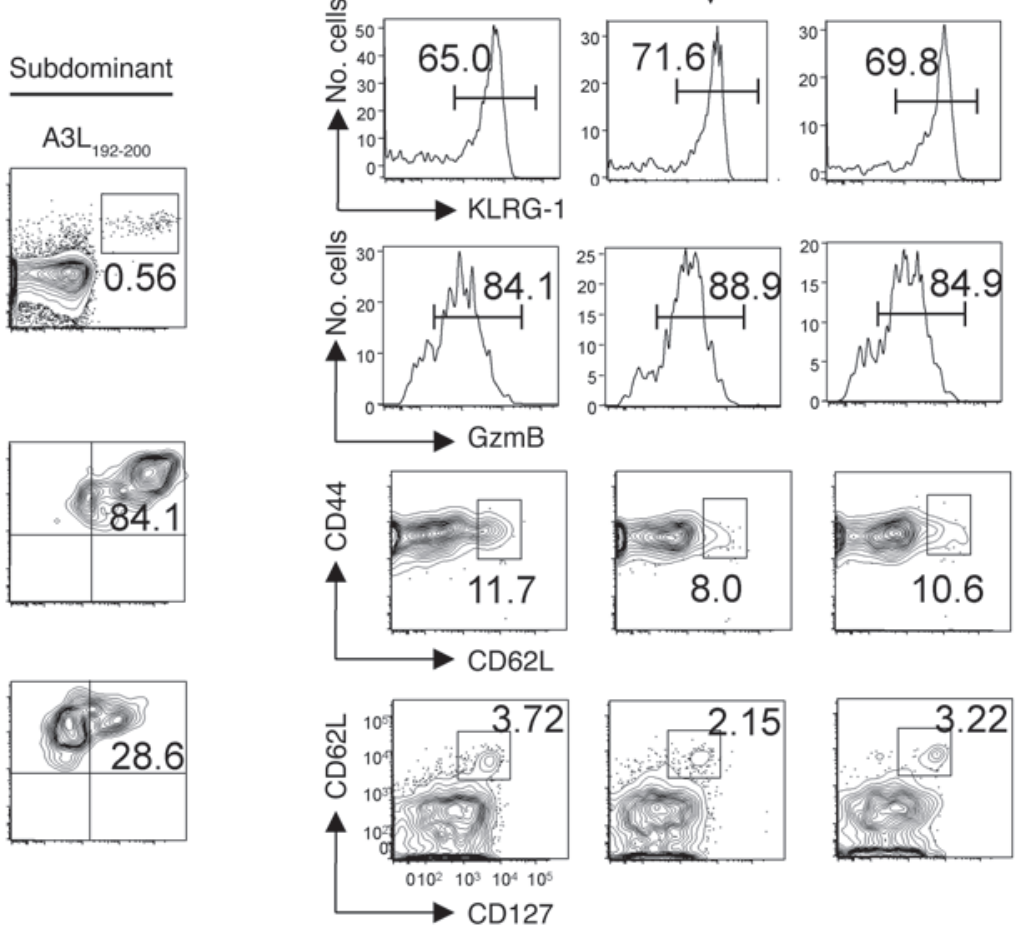

Subdominant

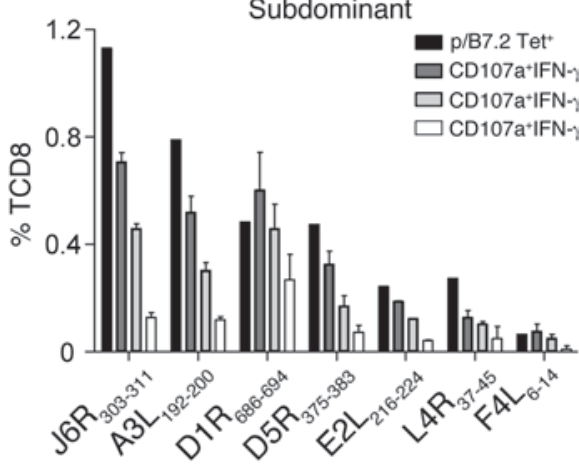

$\mathbf{F}$

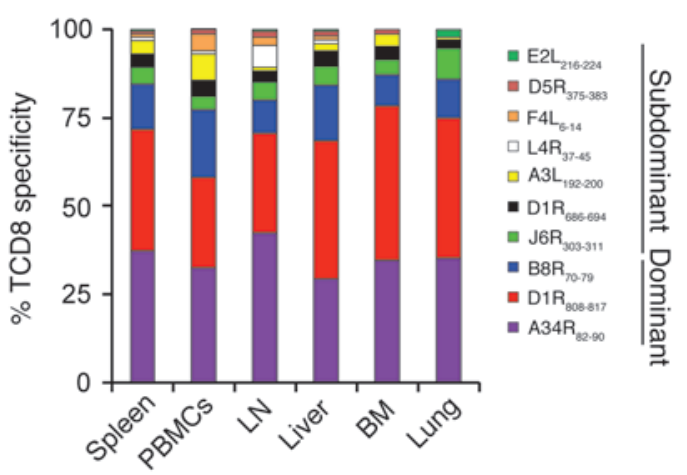




\section{Figure 3}

Individual TCD8 exhibit similar phenotype, functional competence, and homing pattern. (A) Experimental design. B $7^{\text {tg }}$ mice were inoculated i.p. with $2 \times 10^{5}$ pfu VACV, and the TCD8 response was character-

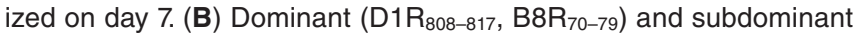
(A3L $\left.{ }_{192-200}\right)$ TCD8 specificities identified in VACV-immune spleens acquired similar effector (CD44hi CD62L LOGzmB+KLRG1hi) and memory (CD44 ${ }^{\text {hi }}$ CD62 $L^{\text {hi }}$ CD127 ${ }^{\text {hi }}$ ) phenotypes. Representative data for 3 TCD8 specificities of 6 measured are shown. Numbers represent the percentage of $p / B 7.2$ tetramer ${ }^{+}$cells within TCD8 gate (top row) or the percentage of p/B7.2 tetramer ${ }^{+}$TCD8 with indicated phenotype. (C and D) Multifunctionality of dominant and subdominant TCD8 detected in immune spleens after ex vivo restimulation with peptide. Data are representative of at least 2 independent experiments. Numbers in $\mathbf{C}$ represent the percentage of IFN- $\gamma^{+} \mathrm{CD} 107 \mathrm{a}^{+}$cells within the TCD8 gate (top row) or the percentage of IFN- $\gamma^{+} \mathrm{CD} 107 \mathrm{a}^{+}$TCD8 expressing the indicated cytokines (middle and bottom rows). Error bars in $\mathbf{D}$ represent mean \pm SD of the assay replicates $(n=3-5)$. (E) Broad TCR V $\beta$ usage within individual TCD8 specificity. Bar segments indicate the proportion of TCR $\beta$ clonotype within the indicated p/B7.2 tetramer+ TCD8 spleen cells. Note that priming with VACV proteins followed by viral challenge enhanced the 4 low-frequency TCD8. (F) Wide tissue distribution of VACV-specific TCD8. Bar segments indicate the proportion of the indicated specificity within the $10 \mathrm{p} / \mathrm{B} 7.2$ tetramer ${ }^{+}$TCD 8 measured. Data are representative of 2 independent experiments $(n=5)$.

TCD8 responses, 1 out of 2 vaccination schemes (Figure 5A) determined above as optimal for a given epitope was applied. We found that nonvaccinated mice were unable to control VACV replication $\left(\sim 10^{8}\right.$ pfu VACV per lungs), and all succumbed to the disease by day 7 (Figure 5, C and E, Supplemental Figure 12). On the other hand, vaccination with 9 out of the 10 tested determinants resulted in various degrees of protection, as judged by disease severity, weight loss, and lung viral load reduction (Figure 5, C-E). Targeting TCD8 against 2 dominant $\left(\mathrm{B}_{8 \mathrm{R}_{70-79},} \mathrm{~A}_{\left.34 \mathrm{R}_{82-90}\right)}\right.$ ) and 4 subdominant $\left(\mathrm{F}_{4} \mathrm{~L}_{6-14}, \mathrm{~A} 3 \mathrm{~L}_{192-200}, \mathrm{~J}_{6} \mathrm{R}_{303-311}, \mathrm{D}_{5} \mathrm{R}_{375-383}\right)$ determinants prevented mortality, with minimal disease and reduced viral titers, although all mice experienced some initial weight loss upon VACV infection (Figure 5, D and E). Notably, B8R $70-79^{-}, \mathrm{A}_{3} \mathrm{~L}_{192-200^{-}}$, and

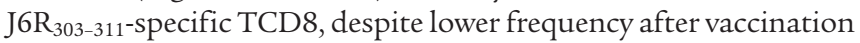
(mean 34.9\%, 22.3\%, and 32.64\%, respectively), protected better than the more frequent $\mathrm{F}_{4} \mathrm{~L}_{6-14}$ (mean $53.6 \%$ ) or $\mathrm{L}_{4} \mathrm{R}_{37-45}$ (mean $86.4 \%$ ) reactive TCD8 specificities (Figure 5, B, D, and E). Nevertheless, the requirement for a higher frequency of responders may explain the insufficient protection conferred by the $\mathrm{D}_{1} \mathrm{R}_{686-694}$, $\mathrm{D}_{1 \mathrm{R}_{808-817}}$, and $\mathrm{E} 2 \mathrm{~L}_{216-224}$ specificities, which were still able to reduce VACV loads in infected lungs (Figure 5, B and C). TCD8 specific to $L 4 R_{37-45}$ were not protective, despite the high frequency of TCD8 present prior to challenge. Despite the very high naive pTCD8 frequency (Figure 4) and their high proliferative potential in response to peptide vaccination (Figure $5 \mathrm{~B}$ ), $\mathrm{L}_{4} \mathrm{R}_{37-45}$ generates a subdominant response in acute VACV infection (Figure 2D). These data suggest that the $\mathrm{L}_{4} \mathrm{R}_{37-45}$ determinant is inefficiently presented during infection (discussed below).

To elucidate the mechanism underlying variable protection conferred by the different naturally processed antigenic peptides, we determined the in vivo presentation kinetics of these TCD8 epitopes. For this, a bulk spleen cell culture was inoculated with VACV (the infection proceeded for 0 to 24 hours) and probed with epitope-specific TCD8 against the 2 most protective determinants

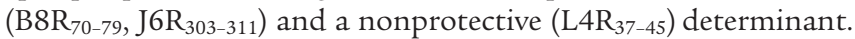
Both protective determinants were displayed on the cell surface for TCD8 appraisal as early as 3 hours, with efficient presentation occurring 24 hours p.i. (Figure 6). In contrast, the nonprotective $\mathrm{L}_{4} \mathrm{R}_{37-45}$ determinant was not efficiently presented during the 24-hour viral infective cycle in vitro (Figure 6). Thus, early and efficient presentation of protective $B 8 \mathrm{R}_{70-79}$ and $\mathrm{J}_{6} \mathrm{R}_{303-311}$ determinants correlated well with their ability to elicit protective TCD8 in vivo.

Finally, we elucidated how TCD8 elicited by epitope vaccination contributed to protection by comparing lung pathology and $\mathrm{T}$ cell infiltration in vaccinated and nonprotected mock-treated mice. Lungs of mock-treated mice were severely damaged, as judged by wide-spread necrosis and fibrin- and edema-filled alveolar spaces (Figure 7A). Vasculitis and perivasculitis of larger pulmonary vessels were also a prominent finding. Inflammatory infiltrates were typically localized around vessels and consisted of neutrophils, macrophages, and few lymphocytes (Figure 7, A and B). Mice vac-

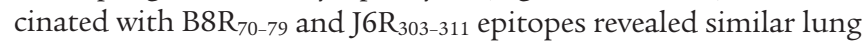
histologic findings, which were distinct from the histopathology of mock-treated mice. These lungs were only moderately damaged (Figure 7A). Within their airways, there were areas of hyperplasia that intersected with areas of necrosis, which were limited to airway epithelium. Airway lumens were filled with sloughed cells, cell debris, macrophages, and few lymphocytes. Infiltrates of lymphocytes formed prominent thick cuffs around airways (Figure 7B). Furthermore, the vast majority of lung TCD8 in protected mice was specific for the vaccine-elicited $B 8 R_{70-79}$ and $J_{6} R_{303-311}$ determinants and produced GzmB (Supplemental Figure 13). These data indicate that efficient presentation of targeted determinants at sites of active viral replication (lungs) ensures robust recruitment and functionality of epitope-specific TCD8 generated by vaccination.

Together our results revealed that the majority of the PTCD8 recognizing naturally processed VACV-derived determinants are capable of evolving into a protective response. In this study, 5 out of the 10 TCD8 specificities generated by vaccination with single antigenic determinants conferred protection, and the remaining 4 specificities significantly reduced viral load in infected lungs despite being nonprotective. Importantly, TCD8 evolved into a protective response regardless of dominance status, suggesting that many PTCD8 specificities recognizing naturally processed epitopes

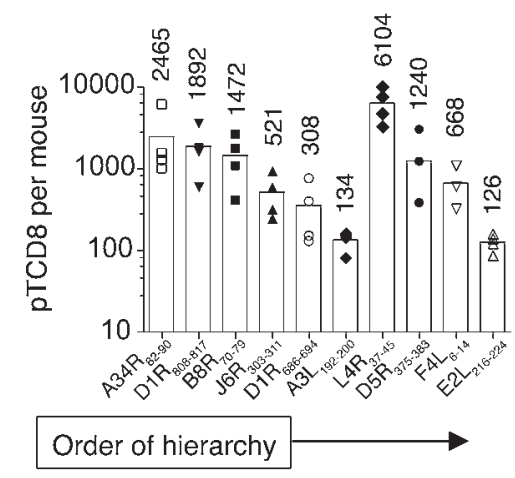

\section{Figure 4}

Frequency of naive pTCD8 that recognize the 10 naturally processed VACV determinants identified in B $7^{\operatorname{tg}}$ mice. Our approach to pTCD8 enumeration is described in Methods and shown in detail in Supplemental Figure 11. Data are representative of 3 to 5 independent experiments using pooled spleen and macroscopic LNs $(n=10)$. Each symbol represents 1 experiment; number indicates mean pTCD8 per mouse. 

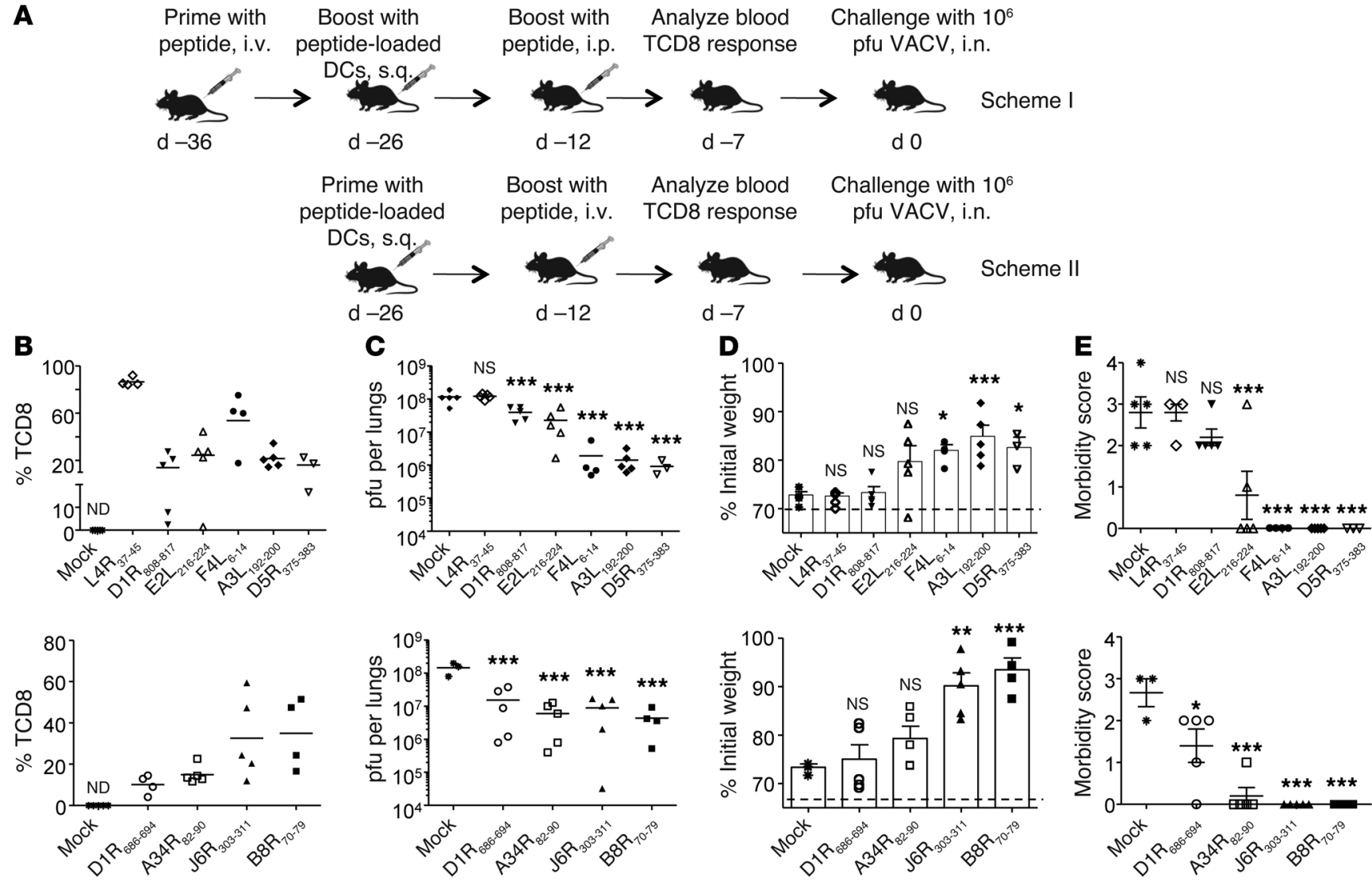

Figure 5

The majority of TCD8 generated against naturally processed determinants confer protective immunity to VACV. (A) Two vaccination schemes. Scheme I entailed priming with peptide formulated with anti-CD40 antibody and poly(I:C), while scheme II entailed priming with epitope-pulsed maturated DCs. (B-E) The top row of panels correspond to peptide vaccination using scheme I; the bottom row of panels correspond to vaccination using scheme II. (B) Epitope-specific TCD8 frequency determined with the indicated p/B7.2 tetramers in blood of vaccinated mice before VACV challenge. nd, not detected. (C) Lung VACV burdens in lethally infected mice evaluated on day 6 p.i. (D) Percentage of initial body weight for epitope-vaccinated mice weighed on day 6 after challenge. The dotted line indicates no recovery threshold (i.e., $\sim 70 \%$ initial body weight). (E) Morbidity score for epitope-vaccinated mice evaluated on day 6 after challenge. Data are representative of 2 independent experiments. Each symbol represents 1 mouse $\left(n=5\right.$ per epitope). Horizontal bars indicate the mean. ${ }^{* \star} P<0.001$; ${ }^{* \star} P<0.01$; ${ }^{\star} P<0.05$; ns, not significant as compared with mock in 1-way ANOVA with Dunnett's post-hoc test. Mean \pm SEM in $\mathbf{D}$ and $\mathbf{E}$ are shown.

may contribute to overall antiviral immunity during acute infection. Our results emphasize the proteomics approach for discovery of naturally processed HLA class I-restricted epitopes that are efficiently presented during infection with a complex pathogen as a viable method to identify targets of protective TCD8 immunity.

\section{Discussion}

The novelty of our work lies in the provision of direct evidence that identification of potent TCD8 targets for pathogens with complex proteomes requires discovery and comprehensive characterization of naturally processed antigenic determinants that are efficiently presented during infection. Hence, in this comprehensive proof-of-principle study, we first discovered naturally processed VACV determinants that are recognized by immune TCD8 and characterized their protective potential in mice. Such an approach in which in-depth insights into fundamental immunobiology of T cell epitopes was used to define targets of protective TCD8 immunity has a broad application, especially for the rational design of TCD8based vaccines against disease agents with complex proteomes.
A large literature supports an essential role for TCD8 in protective immunity against many infectious diseases. Notably, even pathogens with relatively small proteomes, e.g., influenza virus, generate broad TCD8 responses in humans (53), and those with large proteomes, e.g., VACV, elicit a more complex response $(28,29)$. Hence, we would predict the actual breadth of the TCD8 response to complex pathogens - such as Plasmodium or Mycobacterium spp., which surprisingly has never been determined - to be much more complex than those described so far. Moreover, in addition to limited knowledge of immune epitopes, the protective efficacy of individual TCD8 specificities elicited by vaccination has not been elucidated. Consequently, TCD8 protection studies were limited to a few readily detectable epitopes in mice. In contrast, attempts to achieve TCD8-targeted vaccination against complex pathogens in humans were rather empirical and have relied on elicitation of TCD8 with engineered vaccines without solid evidence for efficient presentation of the targeted antigenic determinant during infection $(54,55)$.

Bioinformatics/algorithm-based prediction for TCD8 epitope discovery, a generally accepted approach, resulted in identification 


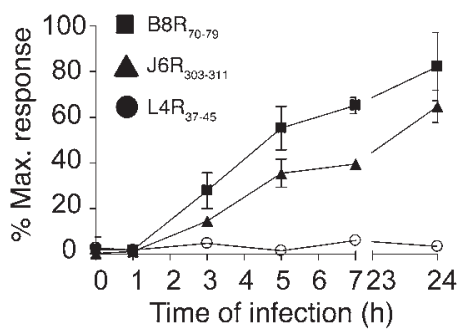

Figure 6

In vitro presentation kinetics of protective and nonprotective immune determinants. Naive spleen cell cultures were inoculated with VACV, and brefeldin $A$ was added to block further presentation of viral peptides at the indicated time points p.i. Infected cells were incubated for 4 hours with ex vivo-expanded TCD8 specific for highly protective $\left(\mathrm{B} \mathrm{R}_{70-79}, \mathrm{~J}_{6} \mathrm{R}_{303-311}\right)$ and nonprotective ( $\left.\mathrm{L}_{4} \mathrm{R}_{37-45}\right)$ epitopes. Antigen presentation at the indicated time points p.i. was assessed by the activation of epitope-specific TCD8, as measured by intracellular IFN- $\gamma$ and TNF- $\alpha$ production. Data are expressed as the percentage of maximal TCD8 stimulation induced by $100 \mathrm{nM}$ peptide. Data are representative of 2 independent experiments performed in duplicate or triplicate (mean $\pm \mathrm{SD}$ ).

of numerous VACV-specific immune determinants. Surprisingly, using our proteomics approach, we identified numerous naturally processed and presented VACV epitopes that were not previously considered as potential TCD8 targets by algorithm prediction and, hence, were not tested for TCD8 recognition. Moreover, by subsequent testing of naturally processed determinants in vaccinated mice and humans, we found many novel immune epitopes, including dominant and subdominant ones. These findings provide evidence that prediction approaches have greatly underestimated the actual breadth of the TCD8 response to VACV. Thus, it is likely that discovery of immune epitopes for pathogens with larger proteomes using algorithms would not be feasible, due to the necessity for screening of large predicted epitope arrays for immune recognition and because currently available T cell assays, such as tetramer staining or ELISpot, albeit sensitive, are severely limit in their potential for high-throughput screening of $\mathrm{T}$ cell epitopes (56).

The sensitivity limits of the proteomics approach favor detection of stably presented and highly abundant MHC-associated deter- minants. Nevertheless, our work suggests that this approach is superior to other approaches because the search for potential vaccine targets is narrowed to those determinants that are efficiently presented during an actual infection. We identified 67 naturally processed B7-restricted antigenic determinants, from which 27 were stably presented by the cognate class I molecule. Ten stably presented determinants were recognized by immune TCD8 in B $7^{\mathrm{tg}}$ mice, together covering nearly $50 \%$ of the overall anti-VACV TCD 8 response. Seven out of the ten epitopes recognized by TCD8 were tested for protection; four of these conferred sufficient protection against lethal respiratory VACV infection. Collectively, approximately $6 \%$ of the eluted peptides, or approximately $15 \%$ of stably presented peptides, were recognized by immune TCD 8 and elicited protective responses in vivo.

The human cell line used as the source of class I-bound peptides may underrepresent or overrepresent actual in vivo breadth of naturally processed and presented VACV determinants. We found one eluted epitope ( $\left.\mathrm{L}_{4} \mathrm{R}_{37-45}\right)$ that was poorly presented during acute VACV infection and generated nonprotective response in epitope vaccination despite the high frequency of functional responders. Some naturally processed determinants of VACV reported previously were not found in this study. It is likely that the use of different cell lines, different multiplicity and kinetics of infection, and engineered soluble class I molecules to elute bound peptides may have resulted in the identification of nonoverlapping naturally processed determinants in different studies $(32,36)$. Some of the qualitative differences mentioned above may have arisen from differences in the activity of the class I peptide-loading complex that associated with soluble and membrane-bound class I molecule. Differences in proteasome activities, both constitutive and immunoproteasomes, in HeLa cells compared with those in professional antigen-presenting cells could have further confounded proteomics-based epitope discovery. Notwithstanding these limitations, because we have functionally characterized the eluted peptides, we believe that this is the first proof-of-principle study suggesting that naturally processed epitopes inform relevant targets for protective TCD8 responses from a microbe with a large proteome.

The protective potential of individual TCD8 specificities elicited during a natural infection or upon successful vaccination has remained underexplored. To elucidate the requirements for

\section{Figure 7}

Epitope-specific TCD8 infiltrated infected lungs and protected them from severe damage. (A) Hematoxylin and eosin stain of infected lungs from nonvaccinated (left) and vaccinated (middle, right) mice. Severe pathology shows wide spread necrosis (no. 1), extensive fibrin deposition (no. 2), edema (no. 3), and vasculitis (no. 4). Moderate damage shows necrosis limited to airway epithelium (no. 5) and signs of regeneration (epithelial hyperplasia; no. 6). (B) Anti-CD3 staining of the infected lungs. Mice vaccinated with $\mathrm{B} \mathrm{R}_{70-79}$ and $\mathrm{J}_{6} \mathrm{R}_{303-311}$ (see Figure 5) show prominent $\mathrm{T}$ cell infiltration around airways, which is present in low numbers in the lungs of mock-vaccinated mice. Scale bar: $25 \mu \mathrm{M}$.
A

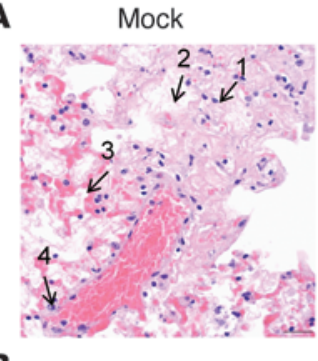

B

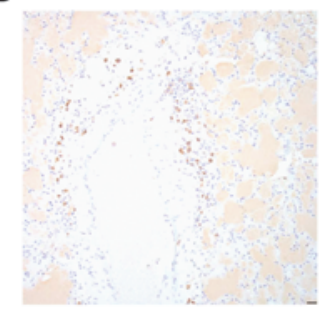

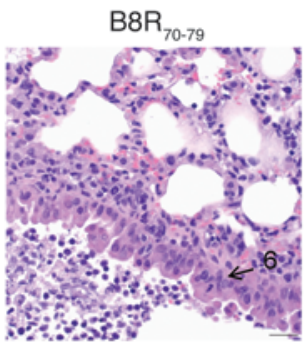
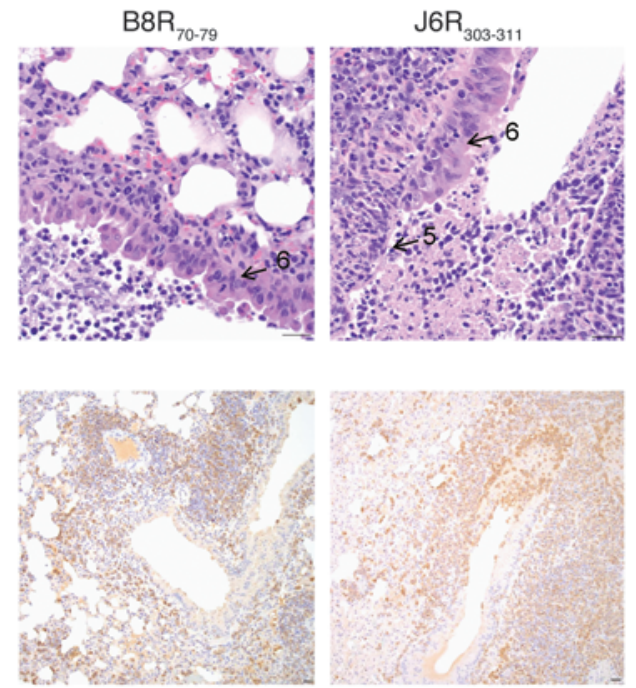
rational targeting of microbial TCD8 epitopes, VACV-immune TCD8 recognizing 10 naturally processed immune determinants of variable dominance were interrogated for phenotype, function, proliferation, and protective efficacy in $\mathrm{B} 7^{\mathrm{tg}}$ mice. Surprisingly, we found that individual TCD8, regardless of their dominance, exhibited similar phenotype and functional competence. Moreover, protective efficacy of individual TCD8 specificities elicited by vaccination was discordant with the established dominance hierarchy. On the other hand, vaccination with a single dominant (B8R $\mathrm{R}_{70-79}$ ) or subdominant $\left(\mathrm{J}_{6} \mathrm{R}_{303-311}\right.$ and $\mathrm{A} 3 \mathrm{~L}_{192-200}$ ) determinant conferred sufficient protection from lethal respiratory VACV infection. Hence, naive precursors of both dominant and subdominant specificities efficiently evolved into multifunctional and protective immune responses upon epitope-targeted vaccination. These results provide the first comprehensive evidence to our knowledge that TCD8 dominance in acute infection does not inform the quality of the immune responders that confer protection. It rather reflects quantitative differences in the frequency of their naive precursors, as was noted by others as well (57). Importantly, we have shown that protection is achieved when targeted immune determinants are efficiently presented during viral infective cycle.

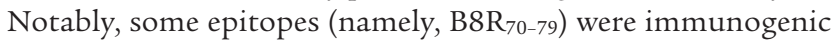
when mice were vaccinated with peptide-pulsed DCs but not with poly(I:C) plus anti-CD40 antibody, suggesting that the immunogenicity of epitopes depends on the form in which they are presented. In one instance, immunization scheme I - in which priming was performed with peptide formulated with poly(I:C) plus anti-CD40 antibody - resulted in a much more robust TCD8 response against $\mathrm{F}_{4} \mathrm{~L}_{6-14}$ than that resulting from scheme II. Consequently, the former resulted in much better protection than the latter, which was essentially nonprotective (data not shown). Therefore, the vaccination scheme itself does not explain the difference in protection. Instead, we found that, to confer protection against lethal VACV challenge, it was critical to achieve a robust TCD8 response by epitope vaccination. Moreover, when immunogenic, we also discovered that individual TCD8 specificities, despite similar functional competence, exhibited varied protective efficacy in active VACV infection. Critically, this varied protection was not directly linked to TCD8 frequency elicited by vaccination: e.g., some responders at lower frequency $\left(\mathrm{A}_{3} \mathrm{~L}_{192-200}\right)$ were more protective than others elicited at higher frequency $\left(\mathrm{F}_{4} \mathrm{~L}_{6-14}\right)$, whereas some responders ( $\left.\mathrm{L}_{4} \mathrm{R}_{37-45}\right)$ were not protective regardless of their frequency. It is likely that differential abundance of determinants and/or kinetics of their natural presentation define visibility of infected cells to cognate TCD8 in vivo, thus restricting protective responses to specificities that efficiently recognize infected cells. For example, we demonstrated that targeting the poorly presented immune determinant $\mathrm{L}_{4} \mathrm{R}_{37-45}$ failed to protect.

Taken together, our findings emphasize the discovery of naturally processed and efficiently presented immune determinants as a rational approach for the identification of protective TCD8 specificities from complex microbial proteomes. Importantly, such protective immune determinants - especially because some of these were commonly recognized by mouse and human

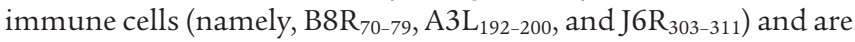
highly conserved among poxviral, including variola, proteomes are potential candidates for TCD8-targeted vaccine design. Application of the approach described herein could benefit discovery of vaccine targets against other human scourges such as malaria, tuberculosis, and HIV/AIDS.

\section{Methods}

Viruses. The Western Reserve strain of VACV (ATCC, VR-119) was grown in and titrated with BSC-40 cells.

Large-scale cell culture and VACV infection. Soluble HLA class I (sA2.1 and sB7.2) production and harvest were as described previously (58). Briefly, approximately $1 \times 10^{9}$ viable cells were inoculated with VACV (MOI 0.1). Supernatants containing approximately $0.3-4.2 \mathrm{mg} / 1 \mathrm{sA} 2.1$ and sB7.2 were collected at 24,48 , and 72 hours after inoculation.

Isolation and fractionation of class I-associated peptides. SA2 and sB7 were affinity purified using W6/32-bound protein A-Sepharose (GE Healthcare). Class I-associated peptide elution, separation, and reversed-phase HPLC purification were all performed as previously described (59).

Mass spectrometry sequencing of eluted peptides. Lyophilized fractions were resuspended in $0.1 \%$ formic acid and subjected to reversed-phase microcapillary LC-nanoESI-MS/MS analysis using an Agilent 1100 binary HPLC pump and an LTQ linear ion trap mass spectrometer 2.2 (Thermo Fisher). A fritless, microcapillary column (100- $\mu \mathrm{m}$ inner diameter) was packed with $10 \mathrm{~cm}$ of $5-\mu \mathrm{m} \mathrm{C}_{18}$ reversed-phase material (Synergi $4 \mu$ Hydro RP80a, Phenomenex) as previously described (60). Reversed-phase HPLC-fractionated peptides were loaded onto the column equilibrated in buffer $\mathrm{A}$ ( $0.1 \%$ formic acid, $5 \%$ acetonitrile) using a LCPackings autosampler. Flow splitting was used to reduce the HPLC flow rate from $200 \mu \mathrm{l}$ per minute to $0.3 \mu \mathrm{l}$ per minute as previously described $(60,61)$. Peptides from the microcapillary column were eluted directly into the linear ion LTQ mass spectrometer equipped with a microelectrospray source (James Hill Instrument Service). Peptides were eluted using a 60-minute linear gradient from $0 \%$ to $60 \%$ buffer B ( $0.1 \%$ formic acid, $80 \%$ acetonitrile) at a flow rate of $0.3 \mu \mathrm{l}$ per minute. During the gradient, the eluted ions were analyzed by 1 full precursor MS scan (400-2,000 m/z), followed by $5 \mathrm{MS} / \mathrm{MS}$ scans of the 5 most abundant ions detected in the precursor MS scan while operating under dynamic exclusion. The program extractms 2 was used to generate the ASCII peak list and identify +1 or multiply charged precursor ions from the native mass spectrometry data file (62) Tandem spectra were searched with no protease specificity using SEQUEST-PVM (63) against a concatenated Human RefSeq protein database release (May 2005) (28,818 entries) and Vaccinia_WR_Copehagen protein database (760 entries). For multiply charged precursor ions $(z \geq+2)$, an independent search was performed on both the +2 and +3 mass of the parent ion. A weighted scoring matrix was used to select the most likely charge state of multiply charged precursor ions as previously described $(64,65)$. Data were processed and organized using the BIGCAT software analysis suite (64).

Bioinformatics. Candidate immune epitopes were selected based solely on their spectral characteristics, namely, (a) their presence in replicate samples (A2) or at multiple time points (A2 and B7); (b) high Cn score; and (c) independent visual confirmation of the mass spectrum. A search for public immune epitopes was conducted through the Immune Epitope Database (http://www.iedb.org/). Peptide conservation among other Orthopoxviridae members was performed using netBLAST search (NCBI).

Volunteers. Individuals (Supplemental Table 2) receiving the DryVax smallpox vaccine were identified and recruited into IRB-approved studies by S. Joyce or C. Oseroff and A. Sette (28). Peripheral blood was obtained by venipuncture, and PBMCs were isolated by centrifugation over FicollPaque (GE Healthcare).

Mice and infection. $\mathrm{B} 7^{\mathrm{tg}}$ and $\mathrm{A} 2^{\mathrm{tg}}$ transgenic mice were previously described (66). These mice do not express the class Ia molecules $\mathrm{H} 2 \mathrm{~K}^{\mathrm{b}}$ and $\mathrm{D}^{\mathrm{b}}$. To elicit primary TCD8 responses, 6- to 8-week-old mice were inoculated i.p. with approximately $2 \times 10^{5} \mathrm{pfu}$ VACV in sterile PBS. After 7 days, mononuclear cells were harvested from the various tissues and used for functional assays.

ELISpot. Ten thousand to 300,000 cells were cocultivated with the appropriate peptide concentration or medium alone for 16 to 18 hours. IFN- $\gamma$ 
secreted into the medium was quantified by ELISpot assay, as previously described, using 1-1.5 $\mu \mathrm{g} / \mathrm{ml}$ anti-human IFN- $\gamma$ (1-D1K, Mabtech) or antimouse IFN- $\gamma$ (AN18, eBioscience) (67).

Statistics. ELISpot data were processed using Statistica software (StatSoft). Where indicated, data comparisons were performed using Prism version 4.0 (GraphPad Software). Multiple group comparisons were performed using 1-way ANOVA with Dunnett's post-hoc test to compare epitope-vaccinated groups with mock. Comparisons between 2 groups were performed using Mann-Whitney test. $P<0.05$ was considered significant.

Study approval. All mice were bred, maintained, and used in experiments in compliance with approved Institutional Animal Care and Use Committee regulations at Vanderbilt University. The Vanderbilt University and La Jolla Institute for Allergy and Immunology IRBs approved all studies involving human PBMCs. All volunteers provided consent per IRB protocol prior to blood collection.

Supplemental material. Additional data are available in the Supplemental Methods, supplemental tables, and supplemental figures, which are available online.

\section{Acknowledgments}

We dedicate this work to the memory of late Professor Stanley G. Nathenson whose numerous pioneering works laid foundation to this line of research. We thank J.S. Bezbradica and Luc Van Kaer for critical reading and advice on the manuscript as well as J.W. Yewdell for helpful discussions on the project. This work was supported by contracts (NO1 AI040079, AI0400024, AI025462), research grants (HL054977, AI042284, AI061721), and core grants (CA068485, DK058404, RR024975) from the NIH and the NIAID Intramural Program.

Received for publication October 17, 2012, and accepted in revised form February 7, 2013.

Address correspondence to: Sebastian Joyce, Vanderbilt University School of Medicine, Department of Pathology, Microbiology and Immunology, A4223 Medical Centre North, 1161 21st Avenue South, Nashville, Tennessee 37232, USA. Phone: 615.322.1472; Fax: 615.343.7392; E-mail: sebastian.joyce@vanderbilt.edu.
1. Zepp F. Principles of vaccine design-Lessons from nature. Vaccine. 2010;28(suppl 3):C14-C24.

2. Amanna IJ, Slifka MK. Wanted, dead or alive: new viral vaccines. Antiviral Res. 2009;84(2):119-130.

3. Golden JW, Hooper JW. The strategic use of novel smallpox vaccines in the post-eradication world. Expert Rev Vaccines. 2011;10(7):1021-1035.

4. Amanna IJ, Slifka MK. Contributions of humoral and cellular immunity to vaccine-induced protection in humans. Virology. 2011;411(2):206-215.

5. Bevan MJ. Understand memory, design better vaccines. Nat Immunol. 2011;12(6):463-465.

6. Pulendran B, Ahmed R. Immunological mechanisms of vaccination. Nat Immunol. 2011;12(6):509-517.

7. Thomas PG, Keating R, Hulse-Post DJ, Doherty PC. Cell-mediated protection in influenza infection. Emerg Infect Dis. 2006;12(1):48-54.

8. Brown LE, Kelso A. Prospects for an influenza vaccine that induces cross-protective cytotoxic T lymphocytes. Immunol Cell Biol. 2009;87(4):300-308.

9. Kohlmeier JE, Woodland DL. Immunity to respiratory viruses. Annu Rev Immunol. 2009;27:61-82.

10. Kremer M, et al. Critical role of perforin-dependent CD8+ T cell immunity for rapid protective vaccination in a murine model for human smallpox. PLoS Pathog. 2012;8(3):e1002557.

11. Goulding J, Bogue R, Tahiliani V, Croft M, Salek-Ardakani S. CD8 T cells are essential for recovery from a respiratory vaccinia virus infection. J Immunol. 2012;189(5):2432-2440.

12. Li CR, Greenberg PD, Gilbert MJ, Goodrich JM, Riddell SR. Recovery of HLA-restricted cytomegalovirus (CMV)-specific T-cell responses after allogeneic bone marrow transplant: correlation with CMV disease and effect of ganciclovir prophylaxis. Blood. 1994;83(7):1971-1979.

13. Goulder PJR, Watkins DI. Impact of MHC class I diversity on immune control of immunodeficiency virus replication. Nat Rev Immunol. 2008;8(8):619-630.

14. Schmidt NW, Butler NS, Badovinac VP, Harty JT. Extreme CD8 T cell requirements for anti-malarial liver-stage immunity following immunization with radiation attenuated sporozoites. Plos Pathogens. 2010;6(7):e1000998.

15. Epstein JE, et al. Live attenuated malaria vaccine designed to protect through hepatic $\mathrm{CD}^{+} \mathrm{T}$ cell immunity. Science. 2011;334(6055):475-480.

16. Woodworth JSM, Behar SM. Mycobacterium tuberculosis-specific CD8(+) T cells and their role in immunity. Crit Rev Immunol. 2006;26(4):317-352.

17. Woodworth JS, Wu Y, Behar SM. Mycobacterium tuberculosis-specific CD8+ T cells require perforin to kill target cells and provide protection in vivo. J Immunol. 2008;181(12):8595-8603.

18. Gordon SN, et al. Smallpox vaccine safety is dependent on T cells and not B cells. J Infect Dis. 2011; 203(8):1043-1053.

19. O'Connell CJ, Karzon DT, Barron AL, Plaut ME, Ali VM. Progressive vaccinia with normal antibodies. a case possibly due to deficient cellular immunity. Ann Intern Med. 1964;60:282-289.

20. Sette A, Rappuoli R. Reverse vaccinology: developing vaccines in the era of genomics. Immunity. 2010; 33(4):530-541.

21. Hel Z, et al. Containment of simian immunodeficiency virus infection in vaccinated macaques: Correlation with the magnitude of virus-specific pre- and postchallenge CD4(+) and CD8(+) T cell responses. J Immunol. 2002;169(9):4778-4787.

22. Moutaftsi $M$, et al. Correlates of protection efficacy induced by vaccinia virus-specific CD8+ T-cell epitopes in the murine intranasal challenge model. Eur J Immunol. 2009;39(3):717-722.

23. Harndahl M, et al. Peptide-MHC class I stability is a better predictor than peptide affinity of CTL immunogenicity. Eur J Immunol. 2012;42(6):1405-1416.

24. Gottschalk RA, et al. Distinct influences of peptide-MHC quality and quantity on in vivo T-cell responses. Proc Natl Acad Sci U S A. 2012; 109(3):881-886.

25. Darrah PA, et al. Multifunctional TH1 cells define a correlate of vaccine-mediated protection against Leishmania major. Nat Med. 2007;13(7):843-850.

26. Araki K, et al. mTOR regulates memory CD8 T-cell differentiation. Nature. 2009;460(7251):108-112.

27. Pasquetto V, et al. HLA-A*0201, HLA-A* 1101 , and HLA-B*0702 transgenic mice recognize numerous poxvirus determinants from a wide variety of viral gene products. J Immunol. 2005;175(8):5504-5515.

28. Oseroff C, et al. HLA class I-restricted responses to vaccinia recognize a broad array of proteins mainly involved in virulence and viral gene regulation. Proc Natl Acad Sci U S A. 2005;102(39):13980-13985.

29. Terajima M, et al. Vaccinia virus-specific CD8(+) $\mathrm{T}$-cell responses target a group of epitopes without a strong immunodominance hierarchy in humans. Hum Immunol. 2008;69(12):815-825.

30. Moutaftsi $\mathrm{M}$, et al. A consensus epitope prediction approach identifies the breadth of murine $\mathrm{T}(\mathrm{CD} 8+)$ cell responses to vaccinia virus. Nat Biotechnol. 2006; 24(7):817-819.

31. Tscharke DC, et al. Identification of poxvirus CD8+ $\mathrm{T}$ cell determinants to enable rational design and characterization of smallpox vaccines. J Exp Med.
2005;201(1):95-104.

32. Meyer VS, et al. Long-term immunity against actual poxviral HLA ligands as identified by differential stable isotope labeling. J Immunol. 2008; 181(9):6371-6383.

33. Snyder JT, Belyakov IM, Dzutsev A, Lemonnier F, Berzofsky JA. Protection against lethal vaccinia virus challenge in HLA-A2 transgenic mice by immunization with a single CD8+ T-cell peptide epitope of vaccinia and variola viruses. J Virol. 2004; 78(13):7052-7060.

34. Remakus S, Rubio D, Ma X, Sette A, Sigal LJ. Memory CD8+ T cells specific for a single immunodominant or subdominant determinant induced by peptide-dendritic cell immunization protect from an acute lethal viral disease. J Virol. 2012; 86(18):9748-9759.

35. Yuen TJ, et al. Analysis of A47, an immunoprevalent protein of vaccinia virus, leads to a reevaluation of the total antiviral CD8+ T cell response. J Virol. 2010;84(19):10220-10229.

36. Johnson KL, Ovsyannikova IG, Mason CJ, Bergen HR. Discovery of naturally processed and HLA-presented class I peptides from vaccinia virus infection using mass spectrometry for vaccine development. Vaccine. 2009;28(1):38-47.

37. Lorente E, et al. Multiple viral ligands naturally presented by different class I molecules in transporter antigen processing-deficient vaccinia virus-infected cells. J Virol. 2012;86(1):527-541.

38. Yewdell JW. Confronting complexity: real-world immunodominance in antiviral CD8 $+\mathrm{T}$ cell responses. Immunity. 2006;25(4):533-543.

39. Hammarlund E, et al. Duration of antiviral immunity after smallpox vaccination. Nat Med. 2003; 9(9):1131-1137.

40. Miller JD, et al. Human effector and memory CD8+ T cell responses to smallpox and yellow fever vaccines. Immunity. 2008;28(5):710-722.

41. Yewdell JW, Bennink JR. Immunodominance in major histocompatibility complex class I-restricted T lymphocyte responses. Annu Rev Immunol. 1999; 17:51-88.

42. Fujii S, Shimizu K, Smith C, Bonifaz L, Steinman RM. Activation of natural killer T cells by alphagalactosylceramide rapidly induces the full maturation of dendritic cells in vivo and thereby acts as an adjuvant for combined CD4 and CD8 T cell immunity to a coadministered protein. J Exp Med. 2003; 198(2):267-279.

43. Giaccone $\mathrm{G}$, et al. A phase I study of the natural killer T-cell ligand alpha-galactosylceramide 
(KRN7000) in patients with solid tumors. Clin Cancer Res. 2002;8(12):3702-3709.

44. Semmling V, et al. Alternative cross-priming through CCL17-CCR4-mediated attraction of CTLs toward NKT cell-licensed DCs. Nat Immunol. 2010; 11(4):313-320.

45. Cerundolo V, Silk JD, Masri SH, Salio M. Harnessing invariant NKT cells in vaccination strategies. Nat Rev Immunol. 2009;9(1):28-38.

46. Lindenstrom T, et al. Tuberculosis subunit vaccination provides long-term protective immunity characterized by multifunctional CD4 memory T cells. J Immunol. 2009;182(12):8047-8055.

47. Flesch IE, et al. Altered CD8(+) T cell immunodominance after vaccinia virus infection and the naive repertoire in inbred and $\mathrm{F}(1)$ mice. J Immunol. 2010; 184(1):45-55.

48. Hamilton SE, Schenkel JM, Akue AD, Jameson SC. IL-2 complex treatment can protect naive mice from bacterial and viral infection. J Immunol. 2010; 185(11):6584-6590.

49. Haluszczak C, et al. The antigen-specific CD8+ $T$ cell repertoire in unimmunized mice includes memory phenotype cells bearing markers of homeostatic expansion. J Exp Med. 2009 206(2):435-448.

50. Akue AD, Lee JY, Jameson SC. Derivation and maintenance of virtual memory CD8 T cells. J Immunol. 2012;188(6):2516-2523.

51. Jenkins MK, Moon JJ. The role of naive T cell precursor frequency and recruitment in dictating immune response magnitude. J Immunol. 2012; 188(9):4135-4140.

52. Cho HI, Celis E. Optimized peptide vaccines eliciting extensive CD8 T-cell responses with therapeutic antitumor effects. Cancer Res. 2009;69(23):9012-9019.

53. Gianfrani C, Oseroff C, Sidney J, Chesnut RW, Sette A. Human memory CTL response specific for influenza A virus is broad and multispecific. Hum Immunol. 2000;61(5):438-452.

54. Sun $\mathrm{P}$, et al. Protective immunity induced with malaria vaccine, RTS,S, is linked to Plasmodium falciparum circumsporozoite protein-specific CD4+ and CD8+ T cells producing IFN-gamma. J Immunol. 2003;171(12):6961-6967.

55. Pinder $\mathrm{M}$, et al. Cellular immunity induced by the recombinant Plasmodium falciparum malaria vaccine, RTS,S/AS02, in semi-immune adults in The Gambia. Clin Exp Immunol. 2004;135(2):286-293.

56. Hadrup SR, et al. Parallel detection of antigen-specific T-cell responses by multidimensional encoding of MHC multimers. Nat Methods. 2009; 6(7):520-526

57. Moon JJ, et al. Naive CD4(+) T cell frequency varies for different epitopes and predicts repertoire diversity and response magnitude. Immunity. 2007 27(2):203-213

58. Prilliman K, Lindsey M, Zuo Y, Jackson KW, Zhang Y, Hildebrand W. Large-scale production of class I bound peptides: assigning a signature to HLA$\mathrm{B} * 1501$. Immunogenetics. 1997;45(6):379-385.

59. Joyce S, Tabaczewski P, Angeletti RH, Nathenson SG, Stroynowski I. A nonpolymorphic major histocompatibility complex class Ib molecule binds a large array of diverse self-peptides. J Exp Med. 1994; 179(2):579-588.

60. Link AJ, LaBaer J, Cold Spring Harbor Laboratory. Proteomics: a Cold Spring Harbor Laboratory Course
Manual. Cold Spring Harbor, New York, USA: Cold Spring Harbor Laboratory Press; 2009.

61. Link AJ, Jennings JL, Washburn MP. Analysis of protein composition using multidimensional chromatography and mass spectrometry. Curr Protoc Protein Sci Chapter. 2004;23:Unit 23.21.

62. Gerbasi VR, Link AJ. The myotonic dystrophy type 2 protein ZNF9 is part of an ITAF complex that promotes cap-independent translation. Mol Cell Proteomics. 2007;6(6):1049-1058.

63. Sadygov RG, et al. Code developments to improve the efficiency of automated MS/MS spectra interpretation. J Proteome Res. 2002;1(3):211-215.

64. McAfee KJ, Duncan DT, Assink M, Link AJ. Analyzing proteomes and protein function using graphical comparative analysis of tandem mass spectrometry results. Mol Cell Proteomics. 2006;5(8):1497-1513.

65. Link AJ, et al. Direct analysis of protein complexes using mass spectrometry. Nat Biotechnol. 1999; 17(7):676-682.

66. Alexander J, Oseroff C, Sidney J, Sette A. Derivation of HLA-B*0702 transgenic mice: functional CTL repertoire and recognition of human $B * 0702$-restricted CTL epitopes. Hum Immunol. 2003; 64(2):211-223.

67. Dragovic SM, et al. Proteasomes, TAP, and endoplasmic reticulum-associated aminopeptidase associated with antigen processing control CD4+ Th cell responses by regulating indirect presentation of MHC class II-restricted cytoplasmic antigens. J Immunol. 2011;186(12):6683-6692.

68. Erickson JJ, et al. Viral acute lower respiratory infections impair CD8+ T cells through PD-1. JClin Invest. 2012;122(8):2967-2982. 